transforms are not degenerate, is quasi-periodic, of period $2 k+1$, its differential equations may be so written that

$$
\begin{gathered}
H_{k-1} ! H_{k} !=1, \quad \mathfrak{A}_{k}{ }^{\prime \prime} \mathfrak{B}_{-k}=H_{k}, \\
\mathfrak{A}_{k}{ }^{\prime \prime} ! H_{k-1} !=\mathfrak{B}_{-(k-1)} .
\end{gathered}
$$

Conversely, any asymptotic plane net, whose $(k+1)$ th and minus $(k+1)$ th transforms are non-degenerate, and for which equations (21) hold, is quasi-periodic, of period $2 k+1$.

ANN Arbor, Mich. April, 1916.

\title{
CONCERNING HILL'S DERIVATION OF THE LAGRANGE EQUATIONS OF MOTION.
}

BY PROFESSOR K. P. WILLIAMS.

(Read before the American Mathematical Society, April 22, 1916.)

THERE are two methods of deriving the Lagrange equations of motion that are commonly given in treatises on dynamics. One of the methods makes use of what is known as Hamilton's principle, while the other proceeds directly from D'Alembert's equation by means of a transformation of variables. While the first method leaves little to be desired as regards elegance, it makes use of a principle not essential to an understanding of the equations of motion or of their application. The second method, as usually given, involves a considerable amount of calculation.

In a paper entitled "On the differential equations of dynamics," in the first volume of the Analyst,* Hill sought to derive the Lagrange equations from D'Alembert's equation without making use of the details of the calculation above mentioned. For some reason his ideas do not seem to have found their way into the literature of the subject. In the form in which he presented it, Hill's derivation seems to me to be open to criticism on account of some of the assumptions that he makes. It is possible, however, to avoid making these assumptions, and when this is done a very simple and direct derivation of the Lagrange equations is obtained.

We start with D'Alembert's equation

* Collected Papers, vol. 1, pp. 192-194. 


$$
\begin{aligned}
\sum_{i=1}^{n}\left[\left(m_{i} \frac{d^{2} x_{i}}{d t^{2}}-X_{i}\right) \delta x_{i}+\left(m_{i} \frac{d^{2} y_{i}}{d t^{2}}-Y_{i}\right) \delta y_{i}\right. \\
\left.+\left(m_{i} \frac{d^{2} z_{i}}{d t^{2}}-Z_{i}\right) \delta z_{i}\right]=0,
\end{aligned}
$$

where $\delta x_{i}, \delta y_{i}, \delta z_{i}$ is any virtual displacement. Since

$$
\frac{d}{d t} \delta u=\delta \frac{d u}{d t} \quad\left(u=x_{i}, y_{i}, z_{i}\right),
$$

we obtain from this in the ordinary way

$$
\text { (1) } \frac{d}{d t} \sum_{i=1}^{n} m_{i}\left\{\frac{d x_{i}}{d t} \delta x_{i}+\frac{d y_{i}}{d t} \delta y_{i}+\frac{d z_{i}}{d t} \delta z_{i}\right\}-\delta(T-U)=0 \text {, }
$$

where $T$ is the kinetic energy and $-U$ the force function. Let now $q_{1}, q_{2}, \cdots, q_{k}$ be the generalized coordinates of the system. Replacing $x_{i}, y_{i}, z_{i}$ by their values in terms of the $q$ 's, the last equation takes the form

$$
\frac{d}{d t} \sum_{i=1}^{k_{k}} p_{i} \delta q_{i}-\delta(\bar{T}-\bar{U})=0
$$

where $p_{1}, p_{2}, \cdots, p_{k}$ are quantities to be determined, and $\bar{T}$ and $\bar{U}$ denote what $T$ and $U$, respectively, become after the substitution. At this point Hill says: "We can find the value of $p_{i}$ without actually making the substitution from this consideration; since the original equation contains only the variations $\delta x, \delta y, \delta z$, etc., without the variations $\delta(d x / d t)$, $\delta(d y / d t), \delta(d z / d t)$, it follows that, in the transformed state, it should contain only the variations $\delta q_{i}$ without the variations $\delta\left(d q_{i} / d t\right)$." It scarcely seems legitimate to draw such a conclusion without further examination, especially since Hill expressly gives to the symbol $\delta(d x / d t)$, etc., no quantitative significance.* The hypothesis that must be made in order to evaluate $p_{i}$ is obtained in the following way.

* The way in which Hill uses the symbol seems to me a little vague. Relative to the relation

$$
\frac{d \delta x}{d t}=\delta \frac{d x}{d t}=\delta x^{\prime}
$$

he states: "The reader will see in this only a notational assumption, without quantitative significance, serving merely as machinery of demonstration." He expressly avoids using $\delta x, \delta y, \delta z$ with the significance attached to them in the calculus of variations. This is legitimate in the case of D'Alembert's equation, where they may represent any virtual displacement, but they must be regarded as certain functions of $t$ in equation (1). 
The last equation when developed becomes

$$
\sum_{i=1}^{k}\left(\frac{d p_{i}}{d t} \delta q_{i}+p_{i} \frac{d}{d t} \delta q_{i}\right)-\sum_{i=1}^{k}\left(\frac{\partial(\bar{T}-\bar{U})}{\partial q_{i}} \delta q_{i}+\frac{\partial \bar{T}}{\partial q_{i}{ }^{\prime}} \delta q^{\prime}{ }^{\prime}\right)=0
$$

or

$$
\text { (2) } \sum_{i=1}^{k}\left[\frac{d p_{i}}{d t}-\frac{\partial(\bar{T}-\bar{U})}{\partial q_{i}}\right] \delta q_{i}+\sum_{i=1}^{k}\left[p_{i}-\frac{\partial \bar{T}}{\partial q_{i}^{\prime}}\right] \frac{d}{d t} \delta q_{i}=0,
$$

since

$$
\delta q_{i}^{\prime}=\frac{d}{d t} \delta q_{i}
$$

Suppose now that the system is holonomic. We can then give to the variations $\delta q_{i}$ any values. Let us make each one of them constant throughout the motion, but not zero. The last summation in the equation above then vanishes, so that we have

$$
\sum_{i=1}^{k}\left[\frac{d p_{i}}{d t}-\frac{\partial(\bar{T}-\bar{U})}{\partial q_{i}}\right] \delta q_{i}=0 .
$$

Since $\delta q_{1}, \cdots, \delta q_{k}$ are all independent we then have at once

$$
\frac{d p_{i}}{d t}-\frac{\partial(\bar{T}-\bar{U})}{\partial q_{i}}=0 \quad(i=1,2, \cdots, k),
$$

an equation entirely independent of the variations $\delta q_{i}$. Equation (2) then becomes

$$
\sum_{i=1}^{k}\left[p_{i}-\frac{\partial \bar{T}}{\partial q_{i}^{\prime}}\right] \frac{d}{d t} \delta q_{i}=0 .
$$

Making all the quantities $\delta q_{i}$ constant except in turn $\delta q_{1}$, $\delta q_{2}, \cdots, \delta q_{k}$, we have finally

$$
p_{i}=\frac{\partial \bar{T}}{\partial q_{i}{ }^{\prime}} .
$$

Upon substituting in (3) we have the Lagrange equations

$$
\frac{d}{d t}\left(\frac{\partial \bar{T}}{\partial q_{i}{ }^{\prime}}\right)-\frac{\partial(\bar{T}-\bar{U})}{\partial q_{i}}=0 \quad(i=1,2, \cdots, k) .
$$

INDIANA UNIVERSITY. 